\title{
Quantum-information entropies for highly excited states of single-particle systems with power-type potentials
}

\author{
J. S. Dehesa* \\ Instituto Carlos I de Física Teórica y Computacional, Granada University, Granada, Spain \\ and Departamento de Física Moderna, Granada University, Granada, Spain \\ A. Martínez-Finkelshtein ${ }^{\dagger}$ \\ University of Almería, Almería, Spain \\ and Instituto Carlos I de Física Teórica y Computacional, Granada University, Granada, Spain
}

V. N. Sorokin

Moscow State University, Moscow, Russia

(Received 7 March 2002; published 20 December 2002)

\begin{abstract}
The asymptotics of the Boltzmann-Shannon information entropy as well as the Renyi entropy for the quantum probability density of a single-particle system with a confining (i.e., bounded below) power-type potential $V(x)=x^{2 k}$ with $k \in \mathbb{N}$ and $x \in \mathbb{R}$, is investigated in the position and momentum spaces within the semiclassical (WKB) approximation. It is found that for highly excited states both physical entropies, as well as their sum, have a logarithmic dependence on its quantum number not only when $k=1$ (harmonic oscillator), but also for any fixed $k$. As a by-product, the extremal case $k \rightarrow \infty$ (the infinite well potential) is also rigorously analyzed. It is shown that not only the position-space entropy has the same constant value for all quantum states, which is a known result, but also that the momentum-space entropy is constant for highly excited states.

DOI: $10.1103 /$ PhysRevA.66.062109

PACS number(s): 03.65.Sq, 03.67.- a, 05.30.-d
\end{abstract}

\section{INTRODUCTION}

The essential reason for the probabilistic character of the quantum theory of physical systems relies upon the uncertainty relation. This relation may be mathematically expressed by means of the Boltzmann-Shannon information entropy (the entropic uncertainty relation) in a much more appropriate and accurate way than by the standard deviation (the Heisenberg or standard uncertainty relation) $[1,2]$. The quantum single-particle probability densities in position and momentum spaces are not necessarily Gaussian or quasiGaussian, but they can take an arbitrary shape; so, in general, the standard deviation is not a useful measure of spreading [3]. The information entropy is an appropriate measure of spreading, and then of quantum uncertainty, a property of fundamental relevance for the adequate characterization of the position and momentum single-particle densities, the basic variables of the modern density-functional theory [4-6] in the two complementary spaces. Additionally, these entropies have been used for numerous practical purposes such as, for example, to measure the squeezing of quantum fluctuation [7] and to reconstruct the charge and momentum densities of atomic and molecular systems [8,9] by means of maximum-entropy procedures.

The analytical determination of the information entropies of physical systems is a formidable project which is now in its infancy. This project has been initiated by the consideration of simple quantum systems: $D$-dimensional single-

\footnotetext{
*Corresponding author. Electronic address: dehesa@ugr.es

†Electronic address: andrei@ual.es

"Electronic address: VNSORMM@nw.math.msu.su
}

particle systems in central potentials. See Ref. [10] for a recent survey, where emphasis is laid on the harmonic oscillator and Coulomb potentials.

The Schrödinger equation of a $D$ dimensional singleparticle system characterized by the Hamiltonian operator $H$ is

$$
H \Psi(\bar{r})=E \Psi(\bar{r}), \quad \bar{r}=\left(x_{1}, \ldots, x_{D}\right),
$$

where $\Psi(\bar{r})$ is the wave function assumed to be normalized to unity. Then, the position density of the system is $\rho(\bar{r})$ $=|\Psi(\bar{r})|^{2}$ and the associated Boltzmann-Shannon information entropy in position space is defined by

$$
S(\rho):=-\int \rho(\bar{r}) \ln \rho(\bar{r}) d \bar{r},
$$

which measures the uncertainty in the spatial localization of the particle [11]. The lower this quantity is, the more concentrated is the wave function, the smaller is the uncertainty, and the higher is the accuracy in predicting the localization of the particle. Analogously, in momentum space the normalized wave function $\hat{\Psi}(\vec{p})$, which is the Fourier transform of $\Psi(\vec{r})$, and its associated Born probability density $\gamma(\vec{p})$ $=|\hat{\Psi}(\vec{p})|^{2}$ has the following information entropy:

$$
S(\gamma):=-\int \gamma(\bar{p}) \ln \gamma(\bar{p}) d \bar{p},
$$

which measures the uncertainty in predicting the momentum of the particle.

These two physical entropies satisfy the inequality

$$
S(\rho)+S(\gamma) \geqslant D(1+\ln \pi),
$$


which is called by entropic uncertainty relation [11-13]. It means that the total uncertainty in position and momentum cannot be decreased beyond the given value $(1+\ln \pi) D$. Nowadays it is well known that this inequality is stronger than the Heisenberg uncertainty relation; see, e.g., Ref. [11].

Most efforts have been concerned with one-dimensional systems $(D=1)$ whose Schrödinger equation,

$$
-\Psi^{\prime \prime}+V(x) \Psi=E \Psi,
$$

has a discrete spectrum of eigenvalues,

$$
E_{0}<E_{1}<E_{2}<\cdots .
$$

Then, the information entropy of the corresponding wave functions $\Psi_{n}(x)$ satisfying the normalization condition,

$$
\int_{\mathbf{R}}\left|\Psi_{n}(x)\right|^{2} d x=1,
$$

will be denoted as

$$
S\left(\Psi_{n}\right):=\int_{\mathbf{R}}\left|\Psi_{n}(x)\right|^{2} \ln \left|\Psi_{n}(x)\right|^{2} d x,
$$

and similarly for the momentum-space information entropy $S\left(\hat{\Psi}_{n}\right)$ of the normalized-to-unity wave function in momentum space $\hat{\Psi}_{n}$. According to Eq. (4), the entropic uncertainty relation for this case reads as

$$
S\left(\Psi_{n}\right)+S\left(\hat{\Psi}_{n}\right) \geqslant 1+\ln \pi,
$$

which indicates that the entropy sum is bounded from below by the value $2.1447 \ldots$.

Recently, it has been shown $[10,14,15]$ that for the harmonic oscillator and Coulomb potentials, the functionals $S\left(\Psi_{n}\right)$ and $S\left(\hat{\Psi}_{n}\right)$ boil down to the integrals of certain classical orthogonal polynomials (Hermite, Laguerre, Gegenbauer) given by

$$
S\left(p_{n}\right):=-\int p_{n}^{2}(x) \ln \left[p_{n}(x)\right]^{2} w(x) d x,
$$

where $p_{n}(x)$ are the polynomials orthogonal with respect to the weight function $w(x)$. These entropylike integrals, which are closely related to the $L^{p}$ norm of the involved polynomials [16], cannot be expressed in a simple form, save for the class of Chebyshev polynomials (which are particular instances of Gegenbauer polynomials) [14,15]. The asymptotics of these integrals, which corresponds to the case $n \rightarrow \infty$, is of special interest for both mathematical and physical reasons. Indeed, the asymptotical behavior of the $L^{p}$ norm of classical orthogonal polynomials is under control [16]. The computation of the asymptotical values of $S\left(p_{n}\right)[10,17]$ opens the way to determine the information entropies of the highly excited (quasiclassical) states as well as to gain insight into the structure and spectroscopy of the recently produced Rydberg atoms [18].

In this paper, we shall analyze the semiclassical asymptotics of the information entropies in position and momen- tum spaces of the wave functions of one-dimensional singleparticle systems with power-type potentials; that is, the eigenfunctions of the wave equation (5) with the potential $V(x)$ given by

$$
V(x)=\mathcal{E}_{k} x^{2 k}, \quad x \in \mathbb{R}, \quad k \in \mathbb{N}, \quad \mathcal{E}_{k}>0
$$

in the framework of the semiclassical (WKB) approximation $[19,20]$. These potentials, which play an important role in quantum field theory and molecular physics, include as special cases the harmonic oscillator $(k=1)$ and the square well $(k \rightarrow \infty)$. They belong to a subclass of potentials $V(x)$ with two turning points, whose position-space information entropy has been recently analyzed [21]; therein it is found a simple relation between the quantum and classical entropies in position space. With an appropriate scaling we may assume that $\mathcal{E}_{k}=1$ in what follows.

It is well known that the spectrum of these smoothly varying potentials consists of a discrete set of eigenvalues (6) for which nontrivial $L^{2}(\mathbb{R})$ solutions $\Psi_{n}$ exist. Notice, in addition, that the Fourier transform $\hat{\Psi}$ of the solution of the Schrödinger equation of these systems,

$$
-\Psi^{\prime \prime}+x^{2 k} \Psi=E \Psi
$$

that is, the momentum wave function

$$
\hat{\Psi}(p)=\frac{1}{\sqrt{2 \pi}} \int_{\mathbf{R}} \Psi(x) e^{-i x p} d x
$$

satisfies the equation

$$
(-1)^{k} \hat{\Psi}^{(2 k)}+p^{2} \hat{\Psi}=E \hat{\Psi} .
$$

We are interested in the behavior when $n \rightarrow \infty$ of the sequences of the position-space entropies $S_{n}=S\left(\Psi_{n}\right)$ and momentum-space entropies $\hat{S}_{n}=S\left(\hat{\Psi}_{n}\right)$ defined in accord with Eq. (8) for the solutions $\Psi_{n}$ and $\hat{\Psi}_{n}$ of Eqs. (12) and (14), respectively, keeping in mind the normalization condition (7) for both functions. For this purpose, we shall follow a two-step procedure that consists in estimating first the $L^{q}$ norm of the corresponding WKB solution and then using the appropriate limit to arrive at the desired asymptotics [16]. This is done in Sec. II for the position-space entropy $S_{n}$ and in Sec. III for the momentum space entropy $\hat{S}_{n}$.

It is found that both physical entropies have a logarithmic dependence on the quantum number $n$ for all the excited states of the semiclassical region. Also, as an important byproduct, the extremal cases $k=1$ (harmonic oscillator) and $k \rightarrow \infty$ (the infinite well) are considered in Sec. IV; in particular, we find the known asymptotical behavior of the position and momentum entropies of the harmonic oscillator $[14,15,17,22]$ and the position entropy of the infinite well [21]. The asymptotics of the momentum entropy of the infinite well potential is also found here without the requirement to any plausible and/or numerical arguments in contrast to a recent work [23]. Finally, let us point out that the asymptot- 
ics of the entropy sum $S_{n}+\hat{S}_{n}$ is explicitly given for the general case as well as for the two special cases mentioned above.

\section{ASYMPTOTICS IN THE POSITION SPACE}

Here the asymptotic behavior of the position-space information entropy $S_{n}=S\left(\Psi_{n}\right)$ of the WKB solutions of the Schrödinger equation (10) of the power-type potential $V(x)$ $=x^{2 k}$ is investigated in detail. For this purpose, we shall estimate first the $L^{q}$ norm of these WKB solutions.

According to the WKB quantization rule $[19,20], E_{n}$ $=E_{n}^{\mathrm{appr}}+o(1)$, where

$$
\frac{1}{\pi} \int \sqrt{E_{n}^{\mathrm{appr}}-x^{2 k}} d x=n+1 / 2, \quad n=0,1,2, \ldots
$$

and the integration is over $x \in \mathbb{R} ; x^{2 k} \leqslant E_{n}^{\text {appr }}$. This relation becomes exact [24] as $n \rightarrow \infty$. See also Ref. [25]. It is straightforward to see that

$$
E_{n}=x_{n}^{2 k}+o(1),
$$

where

$$
x_{n}=\left(\frac{\pi k}{B\left(\frac{3}{2}, \frac{1}{2 k}\right)}(n+1 / 2)\right)^{1 /(1+k)},
$$

and $B(x, y)=\Gamma(x) \Gamma(y) / \Gamma(x+y)$ is the beta function.

If we denote

$$
f_{n}(x)=\sqrt{x_{n}^{2 k}-x^{2 k}}
$$

and

$$
\Phi_{n}(x)=\int_{x}^{x_{n}} f_{n}(t) d t, \quad-x_{n}<x<x_{n},
$$

then the WKB solution of Eq. (5) has the form

$$
\Psi_{n}(x)=C_{n} \frac{\cos \left\{\Phi_{n}(x)-\pi / 4\right\}}{\sqrt{f_{n}(x)}}+o(1),
$$

where $C_{n}$ is a normalization constant. Let us compute the $L^{2 q}$ norm to the power $2 q$ of this function,

$$
N_{n}(q)=\int_{\mathbf{R}}\left|\Psi_{n}(x)\right|^{2 q} d x
$$

From Eq. (17) it is easy to obtain that

$$
\begin{aligned}
N_{n}(q) \sim & \int_{-x_{n}}^{x_{n}}\left|C_{n} \frac{\cos \left\{\Phi_{n}(x)-\pi / 4\right\}}{\sqrt{f_{n}(x)}}\right|^{2 q} d x \\
= & 2 C_{n}^{2 q} x_{n}^{1-k q} \int_{0}^{1}\left|\cos \left[\Phi_{n}\left(x_{n} t\right)-\pi / 4\right]\right|^{2 q} \\
& \times\left(1-t^{2 k}\right)^{-q / 2} d t,
\end{aligned}
$$

where the definition of $f_{n}$ and the substitution $x \mapsto x_{n} t$ have been used. Here and below we write that $a_{n} \sim b_{n}$ when $a_{n}$ $=b_{n}[1+o(1)], n \rightarrow \infty$.

The function

$$
\varphi(t)=\frac{1}{n+1 / 2} \Phi_{n}\left(x_{n} t\right)
$$

is continuous and monotone on $t \in[0,1]$, and $\varphi(t)$ $\in[0, \pi / 2]$. Thus, the inverse function $\omega=\varphi^{-1}$ on $[0, \pi / 2]$ exists and the integral in Eq. (18) is

$$
\int_{0}^{\pi / 2}|\cos [(n+1 / 2) \varphi-\pi / 4]|^{2 q}\left[1-\omega^{2 k}(\varphi)\right]^{-q / 2}\left|\psi^{\prime}(\varphi)\right| d \varphi .
$$

We can use the analog of the Fejer's lemma, established in Lemma 2.1 of Ref. [16], by which this integral tends to

$$
\int_{0}^{\pi / 2}|\cos \varphi|^{2 q} d \varphi \frac{2}{\pi} \int_{0}^{1}\left(1-t^{2 k}\right)^{-q / 2} d t
$$

Thus, by Eq. (18),

$$
N_{n}(q) \sim \frac{x_{n}}{\pi k}\left\{C_{n}^{2} x_{n}^{-k}\right\}^{q} N^{*}(q),
$$

where

$$
N^{*}(q)=B\left(q+\frac{1}{2}, \frac{1}{2}\right) B\left(1-\frac{q}{2}, \frac{1}{2 k}\right) .
$$

Taking $q=1$ and keeping in mind that $N_{n}(1)=1$, we get

$$
1 \sim \frac{B(1 / 2,1 /(2 k))}{2 k} x_{n}^{1-k} C_{n}^{2},
$$

from where the asymptotics of $C_{n}$ follows. Finally, we arrive at

$$
N_{n}(q) \sim \frac{x_{n}}{\pi k}\left\{\frac{2 k}{x_{n}} \frac{1}{B(1 / 2,1 /(2 k))}\right\}^{q} N^{*}(q) .
$$

It is known and easy to verify that the entropy $S_{n}$ is related [16] to the norm $N_{n}(q)$ by

$$
S_{n}=S\left(\Psi_{n}\right)=-\left.\frac{\partial}{\partial q} N_{n}(q)\right|_{q=1}=-\left.\frac{\partial}{\partial q} \ln N_{n}(q)\right|_{q=1} .
$$

Thus,

$$
-S_{n}=\ln \left\{\frac{2 k}{x_{n}} \frac{1}{B(1 / 2,1 /(2 k))}\right\}+\chi+o(1),
$$

where

$$
\chi=\left.\frac{\partial}{\partial q} \ln N^{*}(q)\right|_{q=1} .
$$

We have 


$$
\begin{aligned}
\chi= & \left\{\left[\psi\left(q+\frac{1}{2}\right)-\psi(q+1)\right]\right. \\
& \left.+\frac{1}{2}\left[\psi\left(1+\frac{1}{2 k}-\frac{q}{2}\right)-\psi\left(1-\frac{q}{2}\right)\right]\right\}\left.\right|_{q=1} \\
= & \frac{1}{2}\left[\psi\left(\frac{1}{2}+\frac{1}{2 k}\right)-\psi\left(\frac{1}{2}\right)\right]-\left[\psi(2)-\psi\left(\frac{3}{2}\right)\right],
\end{aligned}
$$

where $\psi(x)=\Gamma^{\prime}(x) / \Gamma(x)$ is the digamma function. With account of Eq. (16),

$$
\begin{aligned}
S_{n}= & \frac{1}{1+k} \ln (2 n)-\frac{1}{2} \frac{1}{1+k} \ln \mathcal{E}_{k}+\frac{k}{1+k} \ln \frac{B(1 / 2,1 /(2 k))}{2 k} \\
& +\frac{1}{1+k} \ln \left(\frac{\pi}{4} \frac{k+1}{k}\right)-\chi+o(1) .
\end{aligned}
$$

Since $\psi(2)-\psi(3 / 2)=2 \ln 2-1$, we finally obtain the following asymptotics for the position-space entropy of a single-particle system submitted to the potential $V(x)=x^{2 k}$, $k \in \mathbb{N}$,

$$
S_{n}=\frac{1}{1+k} \ln (2 n)+\sigma_{k}+o(1), \quad n \rightarrow \infty,
$$

where

$$
\begin{aligned}
\sigma_{k}= & \frac{k}{1+k} \ln \left\{\frac{1}{2 k} B(1 / 2,1 /(2 k))\right\}+\frac{1}{1+k} \ln \left(\frac{\pi}{4} \frac{k+1}{k}\right)+2 \ln 2 \\
& -1-\frac{1}{2}\left[\psi\left(\frac{1}{2}+\frac{1}{2 k}\right)-\psi\left(\frac{1}{2}\right)\right]
\end{aligned}
$$

\section{ASYMPTOTICS IN THE MOMENTUM SPACE}

Here, we shall discuss in detail the asymptotics of the momentum-space information entropy of the WKB momentum wave functions of single-particle system with the powertype potential $V(x)=x^{2 k}, k \in \mathbb{N}$. Once again, we use the WKB method, looking for the solution in the form

$$
\hat{\Psi}(p)=A(p) e^{i S(p)} .
$$

Direct substitution yields that in the first approximation,

$$
\begin{gathered}
\left(S^{\prime}\right)^{2 k}=E-p^{2}, \\
\frac{A^{\prime}}{A}=-\frac{2 k-1}{2} \frac{S^{\prime \prime}}{S^{\prime}} .
\end{gathered}
$$

Thus,

$$
S^{\prime}(p)=\left(E-p^{2}\right)^{1 / 2 k}, \quad A(p)=\left(E-p^{2}\right)^{-(2 k-1) / 4 k}
$$

Denote

$$
g_{n}(p)=\left(E_{n}-p^{2}\right)^{1 / 2 k}, \quad p_{n}=\sqrt{E_{n}} .
$$

Then the oscillating part of $\hat{\Psi}$ is

$$
\hat{\Psi}_{n}(p)=C_{n} \frac{\cos \left\{\Phi_{n}(p)-\pi / 4\right\}}{g_{n}(p)^{(2 k-1) / 2}}+o(1),
$$

where

$$
\Phi_{n}(p)=\int_{p}^{p_{n}} g_{n}(t) d t
$$

The eigenvalue $E_{n}$ is obtained from the Bohr-Sommerfeld quantization rule,

$$
\int_{0}^{E_{n}} g_{n}(t) d t=\frac{\pi}{2}\left(n+\frac{1}{2}\right),
$$

so that

$$
E_{n}=\left(\frac{\pi(n+1 / 2)}{B\left(\frac{1}{2 k}+1, \frac{1}{2}\right)}\right)^{2 k /(1+k)}+o(1),
$$

which agrees with Eq. (15). Now we estimate the norm

$$
\begin{aligned}
N_{n}(q) & =\int_{\mathbf{R}}\left|\hat{\Psi}_{n}(p)\right|^{2 q} d p \\
& \sim \int_{-p_{n}}^{p_{n}}\left|C_{n} \frac{\cos \left\{\Phi_{n}(p)-\pi / 4\right\}}{\left(p_{n}^{2}-p^{2}\right)^{(2 k-1) /(4 k)}}\right|^{2 q} d p .
\end{aligned}
$$

Proceeding as in Eq. (18) we get

$$
N_{n}(q) \sim 2 C_{n}^{2 q} p_{n}^{1-q(2 k-1) / k} \Theta_{n}(q),
$$

with

$$
\begin{aligned}
\Theta_{n}(q)= & \int_{0}^{1}\left|\cos \left[\Phi_{n}\left(p_{n} t\right)-\pi / 4\right]\right|^{2 q}\left(1-t^{2}\right)^{-q(2 k-1) /(2 k)} d t \\
= & \int_{0}^{\pi / 2}|\cos [(n+1 / 2) \omega-\pi / 4]|^{2 q} \\
& \times\left[1-t^{2}(\omega)\right]^{-q(2 k-1) /(2 k)}\left|t^{\prime}(\omega)\right| d \omega,
\end{aligned}
$$

where the substitution $\omega=\Phi_{n}\left(p_{n} t\right) /(n+1 / 2)$ has been used. Again, by Lemma 2.1 in Ref. [6], the last integral tends to

$$
\begin{gathered}
\int_{0}^{\pi / 2}|\cos \omega|^{2 q} d \omega \frac{2}{\pi} \int_{0}^{1}\left(1-t^{2}\right)^{-q(2 k-1) /(2 k)} d t \\
=\frac{1}{2 \pi} B\left(q+\frac{1}{2}, \frac{1}{2}\right) B\left(1-q \frac{2 k-1}{2 k}, \frac{1}{2}\right) .
\end{gathered}
$$


Thus,

$$
\begin{aligned}
N_{n}(q) \sim & \frac{1}{\pi} C_{n}^{2 q} p_{n}^{1-q(2 k-1) / k} B\left(q+\frac{1}{2}, \frac{1}{2}\right) \\
& \times B\left(1-q \frac{2 k-1}{2 k}, \frac{1}{2}\right) .
\end{aligned}
$$

Using the normalization condition $N_{n}(1)=1$, we obtain as above the asymptotics for $C_{n}$,

$$
C_{n} \sim \frac{2 p_{n}^{(k-1) / k}}{B\left(\frac{1}{2 k}, \frac{1}{2}\right)}
$$

Finally,

$$
\begin{aligned}
N_{n}(q) \sim & \frac{1}{\pi}\left\{\frac{2}{B\left(\frac{1}{2 k}, \frac{1}{2}\right)}\right\}^{q} p_{n}^{1-q} B\left(q+\frac{1}{2}, \frac{1}{2}\right) \\
& \times B\left(1-q \frac{2 k-1}{2 k}, \frac{1}{2}\right) .
\end{aligned}
$$

It remains to recall that

$$
\hat{S}_{n}=S\left(\hat{\Psi}_{n}\right)=-\left.\frac{\partial}{\partial q} \ln N_{n}(q)\right|_{q=1},
$$

from where it is straightforward to obtain the asymptotics for the entropy in the momentum space,

$$
\begin{aligned}
\hat{S}_{n}= & \frac{k}{1+k} \ln (2 n)+\frac{1}{1+k} \ln \left\{2 B\left(\frac{1}{2 k}, \frac{1}{2}\right)\right\} \\
& +\frac{k}{1+k} \ln [(k+1) \pi]-1 \\
& +\frac{2 k-1}{2 k}\left[\psi\left(\frac{1}{2 k}\right)-\psi\left(\frac{1}{2 k}+\frac{1}{2}\right)\right]+o(1) .
\end{aligned}
$$

Finally, we can gather the results (21) and (24) in order to find the following estimate for the entropy sum $S\left(\Psi_{n}\right)$ $+S\left(\hat{\Psi}_{n}\right)$ :

$$
\begin{aligned}
S\left(\Psi_{n}\right)+S\left(\hat{\Psi}_{n}\right)= & \ln (2 n)-2-\frac{\gamma}{2}+\ln \left\{\pi \frac{k+1}{k} B\left(\frac{1}{2 k}, \frac{1}{2}\right)\right\} \\
& +\left(1-\frac{1}{2 k}\right) \psi\left(\frac{1}{2 k}\right)-\left(\frac{3}{2}-\frac{1}{2 k}\right) \psi\left(\frac{1}{2 k}+\frac{1}{2}\right) \\
& +o(1),
\end{aligned}
$$

(where $\gamma=0.5772 \ldots$ is the Euler's constant) which certainly fulfils the entropic uncertainty relation (9).

We notice that both position-space and momentum-space entropies as well as their sum increase logarithmically with the quantum number $n$ of the state. The latter is most interesting because of the invariance property of the entropy sum under uniform scaling of the coordinates. Our results show that the level ordering of the power-type potentials is exactly identical for the entropy sum and the single-particle energy.

\section{SPECIAL CASES: HARMONIC OSCILLATOR AND INFINITE WELL}

In this section we particularize the preceding results for the extremal cases: the harmonic oscillator $(k=1)$ and the infinite well $(k \rightarrow \infty)$.

For the harmonic oscillator, one obtains from Eqs. (21) and (24) that both entropies $S\left(\Psi_{n}\right)$ and $S\left(\hat{\Psi}_{n}\right)$ asymptotically behave as

$$
\frac{1}{2} \ln (2 n)+\ln \pi-1,
$$

so that its sum is given by

$$
S\left(\Psi_{n}\right)+S\left(\hat{\Psi}_{n}\right) \sim \ln (2 n)-2+2 \ln \pi
$$

for high values of the quantum number $n$. These results were previously calculated by use of the entropylike integrals of Hermite polynomials $[14,15,17]$ and by the WKB approximation applied to a general one-dimensional potential with two turning points [21]; see also Ref. [22].

Let us now consider the infinite well potential. In this case, the potential $V(x)$ in Eq. (5) becomes

$$
V(x)=\left\{\begin{array}{cc}
0 & \text { if }|x|<1 \\
+\infty & \text { if }|x|>1
\end{array}\right.
$$

Now Eq. (5) describes the motion of a particle confined to an infinite potential well,

$$
-\Psi^{\prime \prime}(x)=E \Psi(x), \quad|x| \leqslant 1, \quad \Psi( \pm 1)=0 .
$$

The solution of this problem is well known,

$$
E_{n}=\left(\frac{\pi n}{2}\right)^{2}, \quad n \in \mathbb{N},
$$

and

$$
\Psi_{n}(x)= \begin{cases}\cos \left(\frac{\pi n x}{2}\right) & \text { if } n \text { is odd } \\ \sin \left(\frac{\pi n x}{2}\right) & \text { if } n \text { is even. }\end{cases}
$$

The corresponding entropy is also easily computed; for $n$ even, 


$$
\begin{aligned}
S\left(\Psi_{n}\right)= & -\int_{-1}^{1} \sin ^{2}(\pi n x) \ln \left[\sin ^{2}(\pi n x)\right] d x \\
= & -\frac{2}{\pi} \int_{0}^{\pi} \sin ^{2} \varphi \ln \left(\sin ^{2} \varphi\right) d \varphi \\
= & -\frac{2}{\pi}\left\{-\frac{\pi}{2} \ln 4+\frac{1}{2 i} \oint_{|z|=1}\right. \\
& \left.\times\left(1-\frac{z}{2}-\frac{1}{2 z}\right) \ln (1-z) \frac{d z}{z}\right\} \\
= & -\frac{2}{\pi}\left\{-\frac{\pi}{2} \ln 4+\frac{\pi}{2}\right\} \\
= & 2 \ln 2-1 .
\end{aligned}
$$

The same result is obtained for the odd values of $n$. Then, the position-space entropy of a particle submitted to an infinite well potential does not depend on the quantum number $n$; that is, it has the same value for all quantum states of the system as already shown $[21,26]$. Therein, it is shown that this quantity is given by

$$
S\left(\Psi_{n}\right)=\ln (4 a)-1
$$

for an infinite well potential defined on the interval $-a \leqslant x$ $\leqslant a$.

We should point out that the entropy for the infinite well case cannot be computed taking formal limit in formulas (21) and (24), because there the $o(1)$ term actually depends on $k$. Nevertheless, it is interesting to observe that the constant term $\sigma_{k}$ in Eq. (22) tends to $2 \ln 2-1$ as $k \rightarrow+\infty$, giving the right answer in the position space.

In the momentum space the wave functions are also well known. Indeed, taking the Fourier transform of Eq. (29) we obtain that

$$
\begin{aligned}
& \hat{\Psi}_{n}(p) \\
& = \begin{cases}(-1)^{(n+1) / 2} \sqrt{2 \pi} \frac{n / 2}{p^{2}-\pi^{2} n^{2} / 4} \cos p & \text { if } n \text { is odd } \\
\frac{(-1)^{n / 2}}{i} \sqrt{2 \pi} \frac{n / 2}{p^{2}-\pi^{2} n^{2} / 4} \sin p & \text { if } n \text { is even. }\end{cases}
\end{aligned}
$$

The entropy in the momentum space for even $n$ (the same result is obtained for the odd values of $n$ ) is

$$
\begin{aligned}
S\left(\hat{\Psi}_{2 n}\right) & =-\int_{\mathbf{R}}\left|\hat{\Psi}_{2 n}(p)\right|^{2} \ln \left|\hat{\Psi}_{2 n}(p)\right|^{2} d p \\
& =-\int_{\mathbf{R}} \mathcal{F}_{n}(x) \ln \left(\frac{1}{\pi n} \mathcal{F}_{n}(x)\right) d x,
\end{aligned}
$$

where the Fejer-type kernel

$$
\mathcal{F}_{n}(x)=\frac{2}{\pi^{2} n} \frac{\sin ^{2}(\pi n x)}{\left(x^{2}-1\right)^{2}}
$$

tends to the half sum of Dirac deltas at $x= \pm 1$. Hence, the momentum-space entropy of a particle in the infinite well potential on the interval $-1 \leqslant x \leqslant 1$ has the value

$$
S\left(\hat{\Psi}_{n}\right)=\ln (2 \pi)+o(1) .
$$

Notice that the result (31) is not recovered by a formal limit $k \rightarrow \infty$ in Eq. (24). For completeness, let us mention that the recent computation of this quantity by Majernik et al. [23] uses an interchange of the order of operations limit and integration [see Eq. (17), p. 2212 of Ref. [23]). As the authors recognize, this is not justified for their integral with Fejertype kernel and variable limits of integration; in fact, this procedure leads to a wrong value of the entropy.

Finally, let us remark that the entropy sum for the infinite well is

$$
S\left(\Psi_{n}\right)+S\left(\hat{\Psi}_{n}\right) \sim 3 \ln 2-1+\ln \pi \geqslant 1+\ln \pi
$$

in compliance with the entropic uncertainty relationship (9) which is valid for any wave function of one-dimensional systems.

\section{SUMMARY, OPEN PROBLEMS, AND CONCLUSION}

We have calculated the position and momentum information entropies for the highly excited states of the power-type potential $V(x)=x^{2 k}$ with integer $k$ by means of the semiclassical (WKB) approximation. Our results show that the position entropy grows as $(k+1)^{-1} \ln n$ and the momentum entropy increases as $k(k+1)^{-1} \ln n$ when the quantum number $n$ characterizing the state tends to infinity. Since the entropy sum is invariant under uniform scaling of the single-particle coordinates, it is most interesting to realize that this quantity, which has the net information content of the system, also increases logarithmically as the quantum number grows. As a consequence, the ordering of the quantum levels is exactly identical for the entropy sum and the single-particle energy. This property of the entropy sum is shared by other physical systems of different dimensionalities such as the twodimensional harmonic oscillator [27] and circular membrane [28], and the neutral atoms $[29,30]$. Then, our results support the idea to develop a new maximum entropy procedure based on the maximization of the entropy sum subject to some known position and momentum constraints, which would extend the Jaynes' maximum entropy method to the case involving constraints in the two complementary spaces. This procedure has been already proposed [31], but still remains largely unexplored.

It is well known that the entropic uncertainty relation (4) is stronger than the Heisenberg uncertainty relation [11], which is closely connected to the fact that the entropic measure of dispersion expresses more adequately the intuitive concept of uncertainty than the variance [32]. Nevertheless, it would be an interesting open problen to calculate the Heisenberg position and momentum uncertainties $\Delta x$ and 
$\Delta p$, and to contrast their behaviors with the aforementioned logarithmic growth of the information entropy in corresponding spaces. On the other hand, we are still looking for a measure that allows us to explain the qualitative property of increasing concentration of the wave function on classical periodic orbits as excitation grows. Which is the measure of concentration which decreases as the quantum number $n$ grows? Might Fisher's measure be a good candidate?

Finally, as a subproduct, we have found the aforementioned entropic quantities for the harmonic oscillator which had been previously calculated by other means $[14,15,17,21,22]$, which confirms our results at the extreme case $k=1$. In addition, we have determined for the infinite well potential (case $k \rightarrow \infty$ ) not only the position entropy, producing the previously known value $[21,26]$ but also the momentum entropy which up to now was not rigorously known in spite of some efforts based on some plausible and/or numerical arguments [23]. Needless to say, that the entropy sum (which is a joint measure of the positionmomentum uncertainty) fulfills the entropic uncertainty relationship (9) for all the systems considered in this work, what is a further check of our calculations.

\section{ACKNOWLEDGMENTS}

We gratefully acknowledge the support by the European Union project INTAS-2000-272 and the Spanish research grant BFM2001-3878-C02 (J.S.D. and A.M.F.) as well as by the Russian Grants Nos. RFFR 96-01-00730 and 96-1596185 (V.N.S.).
[1] J. Uffink and J. Hilgewoord, Found. Phys. 15, 925 (1985).

[2] M. J. W. Hall, Phys. Rev. A 59, 2602 (1999).

[3] M. Ohya and D. Petz, Quantum Entropy and its Use (SpringerVerlag, Berlin, 1993).

[4] P. Hohenberg and W. Kohn, Phys. Rev. A 136, 864 (1964).

[5] R. G. Parr and W. Yang, Density Fuctional Theory of Atoms and Molecule (Oxford University Press, Oxford, 1989).

[6] N. H. March, Electron Density Theory of Atoms and Molecules (Academic, New York, 1992).

[7] A. Orlowski, Phys. Rev. A 56, 2545 (1997).

[8] A. Galindo and P. Pascual, Quantum Mechanics (Springer, Berlin, 1978).

[9] J. C. Angulo, J. Antolín, A. Zarzo, and J. C. Cuchí, Eur. Phys. J. D 7, 479 (1999).

[10] J. S. Dehesa, A. Martínez-Finkelshtein, and J. Sánchez-Ruiz, J. Comput. Appl. Math. 133, 23 (2000).

[11] I. Bialynicki-Birula and J. Mycielski, Commun. Math. Phys. 44, 129 (1975).

[12] W. Beckner, Ann. Math. 102, 159 (1975).

[13] I. Bialynicki-Birula, Phys. Lett. 103A, 253 (1984).

[14] R. J. Yánez, W. Van Assche, and J. S. Dehesa, Phys. Rev. A 50, 3065 (1994).

[15] J. S. Dehesa, W. Van Assche, and R. J. Yáñez, Meth. Appl. Anal. 4, 91 (1997).

[16] A. I. Aptekarev, V. S. Buyarov, and J. S. Dehesa, Russ. Acad. Sci. Sb. Math. 82, 373 (1995).
[17] W. Van Assche, R. J. Yáñez, and J. S. Dehesa, J. Math. Phys. 36, 4106 (1995).

[18] T. F. Gallager, in Atomic, Molecular and Optical Physics: Atoms and Molecules, edited by F. B. Dũnning and R. G. Hulet (Academic, New York, 1996).

[19] L. D. Landau and E. M. Lifshitz, Quantum Mechanics: Nonrelativistic Theory (Pergamon, Oxford, 1977).

[20] V. P. Maslov and M. V. Fedoriuk, Semiclassical Approximations in Quantum Mechanics: I (Reidal, Dordrecht, 1981).

[21] J. Sánchez-Ruiz, Phys. Lett. A 226, 7 (1997).

[22] V. Majerník and T. Opatrný, J. Phys. A 29, 2187 (1996).

[23] V. Majerník, R. Charvot, and E. Majerníková, J. Phys. A 32, 2207 (1999).

[24] C. M. Bender, K. Olausen, and P. S. Wang, Phys. Rev. D 16, 1740 (1977).

[25] A. Voros, J. Phys. A 27, 4653 (1994).

[26] D. A. Bochvar, I. V. Stankevich, and A. L. Chistyakov, Russ. Chem. Rev. 43, 276 (1974).

[27] J. S. Dehesa, R. J. Yáñez, A. I. Aptekarev, and V. Buyarov, J. Math. Phys. 39, 3050 (1998).

[28] J. S. Dehesa, A. Martínez-Finkelshtein, and V. N. Sorokin, Int. J. Bifurcation Chaos Appl. Sci. Eng. 12, 11 (2002).

[29] S. R. Gadre, S. B. Sears, S. J. Chakravorty, and R. D. Bendale, Phys. Rev. A 32, 2602 (1985).

[30] S. R. Gadre and R. D. Bendale, Phys. Rev. A 36, 1932 (1987).

[31] S. R. Gadre and R. D. Bendale, Int. J. Quantum Chem. 28, 311 (1985).

[32] V. Majerník and L. Richterek, Eur. J. Phys. 18, 79 (1997). 